\title{
REC8 suppresses tumor angiogenesis by inhibition of NF-KB-mediated vascular endothelial growth factor expression in gastric cancer cells
}

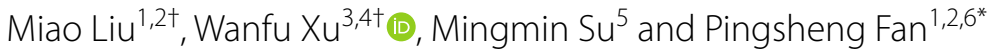

\begin{abstract}
Background: Tumor angiogenesis is an essential event for tumor growth and metastasis. It has been showed that REC8, a component of the meiotic cohesion complex, played a vital role in Epithelial-Mesenchymal Transition (EMT) in gastric cancer. However, the role of REC8 in gastric cancer angiogenesis remains to be identified.

Results: Inhibition of REC8 expression in gastric cancer cells contributed to tumor angiogenesis in the gastric cancer microenvironment. The clinical analysis demonstrated that the loss of REC8 in gastric cancer with enrichment of MVD. Depletion of REC8 expression in gastric cancer cells significantly increased tube formation of human umbilical vein endothelial cells (HUVECs), which is attributed to enhancement of vascular endothelial growth factor (VEGF) secretion caused by REC8 slicing. While addition of neutralizing antibody targeted VEGF into supernatant drastically reversed the effect of REC8 loss in gastric cancer cells on tube formation. Mechanistic analyses indicated that ablation of REC8 promotes nuclear factor-KB (NF-KB) p65 activity and its downstream gene VEGF expression, leading to tube formation.
\end{abstract} Conclusions: These results demonstrated a novel REC8 function that suppressed tumor angiogenesis and progression by attenuation of VEGF in gastric cancer microenvironment.

Keywords: REC8, Tumor angiogenesis, VEGF, Gastric cancer

\section{Background}

Despite tremendous effort has been made to improve the effectiveness of treatment, gastric cancer is a common type of malignant tumor with relatively poor prognosis and presents a serious threat to global health [1], ranking fifth in terms of incidence and third in terms of mortality worldwide [2]. A growing body of evidences indicated that angiogenesis, one of the cancer hallmarks, is key requirement for tumor growth, invasion [3]. Targeting

\footnotetext{
*Correspondence: fanpingsheng@csco.ac.cn

${ }^{\dagger}$ Miao Liu and Wanfu Xu contributed equally to this work

1 Anhui Provincial Hospital, Cheeloo College of Medicine, Shandong University, Jinan 250021, Shandong, China

Full list of author information is available at the end of the article
}

vessel formation and disturbing tumor vasculature have been among the primary strategies in cancer treatment [4]. However, the contribution of tumor angiogenesis remained elusive.

Angiogenesis is defined as the formation of new blood vessels from preexisting vessels and has been characterized as an essential process for tumor cell proliferation and viability [5], while a large number of evidences indicated that VEGF is widely accepted as a primary inducer of angiogenesis [6, 7]. VEGF, binding to its receptors, especially VEGFR2, appeared to be a key factor in pathological situations that involved in tumor neovascularization [8]. Evidence has been shown that inhibitors of VEGF and VEGFR2 reduced endothelial cell proliferation, migration and survival that led to regression

c) The Author(s) 2020. This article is licensed under a Creative Commons Attribution 4.0 International License, which permits use, sharing, adaptation, distribution and reproduction in any medium or format, as long as you give appropriate credit to the original author(s) and the source, provide a link to the Creative Commons licence, and indicate if changes were made. The images or other third party material in this article are included in the article's Creative Commons licence, unless indicated otherwise in a credit line to the material. If material is not included in the article's Creative Commons licence and your intended use is not permitted by statutory regulation or exceeds the permitted use, you will need to obtain permission directly from the copyright holder. To view a copy of this licence, visit http://creativeco mmons.org/licenses/by/4.0/. The Creative Commons Public Domain Dedication waiver (http://creativecommons.org/publicdomain/ zero/1.0/) applies to the data made available in this article, unless otherwise stated in a credit line to the data. 
of vessel density and decrease vascular permeability, thereby slowing tumor growth $[9,10]$. however, the upstream of these transcription factors remained to be fully elucidated.

REC8, a key meiosis-specific component of the cohesion complex, has been implicated in DNA damage repair and maintenance of chromosome stability [11, 12]. It has been reported that REC8 was hypermethylated in melanomas and malignant gastrointestinal stromal tumors [13-15], suggesting the low level of REC8 expression in tumor and a potential tumor suppressor. These findings suggested that the multifaceted REC8-mediated anticancer effects played a causal but unclear role in mammalian oncogenesis. However, there is no available reports about the function of REC8 on tumor angiogenesis. In this study, we are for the first time to demonstrate that REC8 played an anti-angiogenic role in the tumor angiogenesis in gastric cancer and defined a novel model in which REC8 inhibited NF- $\mathrm{kB}$ pathway in gastric cancer cells through suppression of VEGF expression, leading to inhibit angiogenesis. Most importantly, the expression of REC8 level is negatively correlation with MVD in gastric cancer. This finding not only provided further support for the tumor suppressor role of REC8, but also added a novel link between abnormal cell meiosis and tumor angiogenesis.

\section{Materials and methods}

\section{Chemicals and reagents}

The simpleChIP ${ }^{\circledR}$ plus enzymatic chromatin IP Kit was from Cell Signal Technology (Danvers, MA, USA). Allin-One First-Strand cDNA Synthesis Kit and All-in-One qPCR Mix were from GeneCopoeia (Rockville, MD, USA). Antibodies used for immunoblotting and IP assays were as follows: the NF-KB p65 primary antibody for chromatin IP was purchased from CST; primary antibodies against VEGF were from Abclonal (Cat: A17877, Wuhan, China);

\section{Cell lines, cell cultures and transfection}

The gastric cancer cell lines of poor differentiation, BGC823 and AGS-1, human umbilical vein endothelial cells (HUVECs) were obtained from Cell Bank of the Chinese Academy of Sciences (Chinese Academy of Sciences, Shanghai, China) and cultured in Dulbecco's Modified Eagle's Medium (DMEM, Gibco) supplemented with $10 \%(\mathrm{v} / \mathrm{v})$ fetal bovine serum $(\mathrm{FBS})$ at $37^{\circ} \mathrm{C}$ in a humidified atmosphere of $5 \% \mathrm{CO}_{2}$. The plasmids were transfected into cells with Hilymax according to manufacturer's protocol.

\section{Generation of stable cell line}

The lentivirus control shRNA (shCTL: sc-108080) and REC8 shRNA (shREC8: sc-106878-V) were purchased from Santacruz Biotechnology, INC. Puromycin was purchased from sigma and used to select for stably infected cells.

\section{RNA extraction and quantitative real-time PCR}

Total RNA was extracted from cultured cells with trizol reagent (Invitrogen, Carlsbad, CA, USA) according to the manufacturer's protocol. All cDNA samples were prepared using an All-in-one First-stand cDNA synthesis kit (GeneCopoeia, MD, USA). Quantitative real-time PCR (RT-qPCR) analyses were performed with an all-in-one qPCR mix (GeneCopoeia) according to manufacturer's instructions using an ABI StepOne-Plus ${ }^{\mathrm{TM}}$ qPCR system. The primer for VEGF: Forward: $5^{\prime}$-TGCAGATTATGC GGATCAAACC-3'; Reverse: 5'-TGCATTCACATTTGT TGTGCTGTAG-3'; REC8: Forward: 5'-CATCCCACC AGAAGAACGG-3'; Reverse: 5'-GCACCAAAGGCA TCTCCAT-3'; $\beta$-actin: Forward 5'-ATCGTGCGTGAC ATTAAGGAGAAG-3'; reverse:5'-AGGAAGGAAGGC TGGAAGAGTG-3'.

\section{Endothelial cell tube formation assay}

As described in $\mathrm{Xu}$ et al. study [16], 96-well plates were coated with matrigel basement membrane matrix (BD Biosciences) and then allowed to polymerize at $37{ }^{\circ} \mathrm{C}$ for at least $30 \mathrm{~min}$. HUVECs were treated with conditional medium for 6-8 h, tubes formation of HUVECs can be visualized and the number of nodes (defined as when at least three cells formed a single point) per image was quantified as described [17].

\section{Immunoblotting assays}

As described in the study [18], cell lysates were lysed with $2 \times$ loading sample buffer and analyzed by immunoblotting to detect proteins. Briefly, the protein was transferred to a $0.22 \mu \mathrm{m}$ nitrocellulose transfer membrane. The membrane was blocked with $5 \%(\mathrm{w} / \mathrm{v})$ milk in PBS/ $0.05 \%(\mathrm{v} / \mathrm{v})$ Tween-20 and then incubated with the indicated antibody overnight. This was followed by incubation with a horseradish peroxidase secondary antibody (Jackson ImmunoResearch) for $1 \mathrm{~h}$ at room temperature. Proteins were detected using enhanced chemiluminescence substrates (Perkin Elmer). The antibodies listed as followed: REC8(abgent, AP13570c,1:2000 for WB; proteintech 11913-1-AP, 1:200 for IP), VEGF(abclonal, A12303,1:2000 for WB; MAB293, 1:50 for neutralize), NF-kB p65 (abclonal, A11202, 1:2000 for WB), PhosphoNF-кB p65(Ser536) (Ser536) (abclonal, AP0124, 1:2000 for WB). $\alpha$-tubulin (abclonal, AC012); $\beta$-actin (abclonal, AC004). 


\section{Enzyme-linked immunosorbent assays (ELISA)}

Quantitative measurement of VEGF secreted into conditional medium was determined using ELISA according to the manufacturer's protocol (elabscience, E-EL-H0111c).

Immunohistochemistry (IHC) of human tissue microarrays A human gastric cancer tissue microarray was purchased from Alenabio. Gastric cancer samples were immunostained against indicated antibodies as previously described [18]. Briefly, the slides were dewaxed and rehydrated in distilled water, sections were immersed in citrate buffer $\left(\mathrm{C}_{6} \mathrm{H}_{5} \mathrm{Na}_{3} \mathrm{O}_{7} \cdot 2 \mathrm{H}_{2} \mathrm{O}\right)$ and then microwaved for $20 \mathrm{~min}$ for antigen retrieval. Endogenous peroxidase activity was blocked with $0.5 \%(\mathrm{v} / \mathrm{v}) \mathrm{H}_{2} \mathrm{O}_{2}$. The slides were then transferred into a humidified chamber, incubated with $5 \%(\mathrm{v} / \mathrm{v})$ horse serum for $30 \mathrm{~min}$ and then incubated with primary antibodies overnight at $4{ }^{\circ} \mathrm{C}$. After primary antibody CD31(Sangon Biothch, D260721,1:200 for IHC), REC8 (Proteintech 10793-1AP, 1:200 for IHC) incubation, the slides were immersed in peroxidase-labeled secondary antibody for $30 \mathrm{~min}$ at room temperature. To detect the antibody-conjugated antigen reaction, the sections were incubated in 3-amino9-ethylcarbazole substrate-chromogen for $30 \mathrm{~min}$ and counterstained with hematoxylin. The evaluation of the staining was performed as described in our pervious study [19]. Quantitative analysis of the immunostained images of human biopsies was performed by positive cell number counting and computerized optical density (OD) measurements with Image Pro Plus 6.0 software (Media Cybernetics, MD, USA).

\section{Chromatin immunoprecipitation (ChIP) assays}

ChIP assays were performed in BGC823 cells by using a SimpleChIP ${ }^{\circledR}$ Plus Enzymatic Chromatin IP Kit as pervious work [20]. Briefly, $1.0 \times 10^{7}$ cells were crosslinked with $1 \%(\mathrm{w} / \mathrm{v})$ formaldehyde for $10 \mathrm{~min}$ and then quenched in $0.125 \mathrm{M}$ glycine for $5 \mathrm{~min}$. Cells were lysed and digested to collect the chromatin. IP was carried out by using the indicated antibody overnight. The precipitated DNAs were analyzed and quantified by using real-time PCR analysis. Primer sequences for VEGF (NM_001171622) listed as followed: forward 5'-CGT GTGGAAGGGCTGAGG-3', reverse 5'-CCGCTACCA GCCGACTTTT-3',

\section{Statistical analysis}

All statistical analyses and statistical graphing were done using GraphPad Prism 8 software. All the t test was used to determine the significance of differences in the qPCR assay. One-way ANOVA was performed on data from endothelial cell tube formation assay. For correlation analysis, p value of less than 0.05 was considered statistically significant.

\section{Results}

Downregulation of REC8 in GC was negatively correlated with microvascular density.

To further explore the possible clinical relationship between REC8 and microvascular density (MVD), we performed immunohistochemical staining to reveal REC8 expression is extraordinarily reduced in patients with GC $(n=59)$ compared with that in adjacent tissue $(\mathrm{n}=16)$ (Fig. 1a). Inversely, CD31 (an endothelial cell marker) and MVD were significantly increased in GC compared with adjacent tissue and quantified in Fig. $1 \mathrm{~b}$. These results suggested the possible role of REC8 in tumor angiogenesis.

\section{Inhibition of REC8 expression in gastric cancer cells promoted HUVEC cells tube formation}

To explore possible role of REC8 in the tumor angiogenesis, we established BGC823 and AGS-1 cells that stably expressed REC8 depletion by lentivirus infection and confirmed by real-time PCR and WB (Fig. 2a, b). Next, we investigated the effect of REC8 in gastric cancer cells on HUVECs migration using transwell model system. As shown in Fig. 2c, d, the conditional medium (CM) from REC8-depleted BGC823 or AGS-1 cells significantly increased the chemotactic migration of HUVECs compared with CM from the shCTL group. Further investigation showed that supernatant from ablated of REC8 expression in gastric cancer cells drastically enhanced HUVEC tube formation by a co-cultured with HUVECs (Fig. 2e, f). In summary, these results showed that the critical role of REC8 in mediating tumor angiogenesis.

\section{REC8 suppressed tube formation in dependent of VEGF}

It is noteworthy that VEGF secreted by cancer cells acted as chemoattractants in promoting motility of endothelial cell during tumor angiogenesis [21, 22], which focused us to seek the potential role of REC8 on VEGF expression. Interestingly, we found that VEGF expression was significantly increased at mRNA level in BGC823 cells with REC8 knockdown (Fig. 3a), in line with this, WB results reveled that depletion of REC8 expression in BGC823 and AGS-1 cells drastically increased VEGF expression level (Fig. 3b), which is also confirmed by Elisa results (Fig. 3c). What's more, neutralizing VEGF in CM significantly abrogated CM that from REC8-depleted BGC823induced migration of HUVECs (Fig. 3d, e), leading to inhibit angiogenesis (Fig. 3f, g), implying that REC8 facilitated angiogenesis at least in part by triggering the 


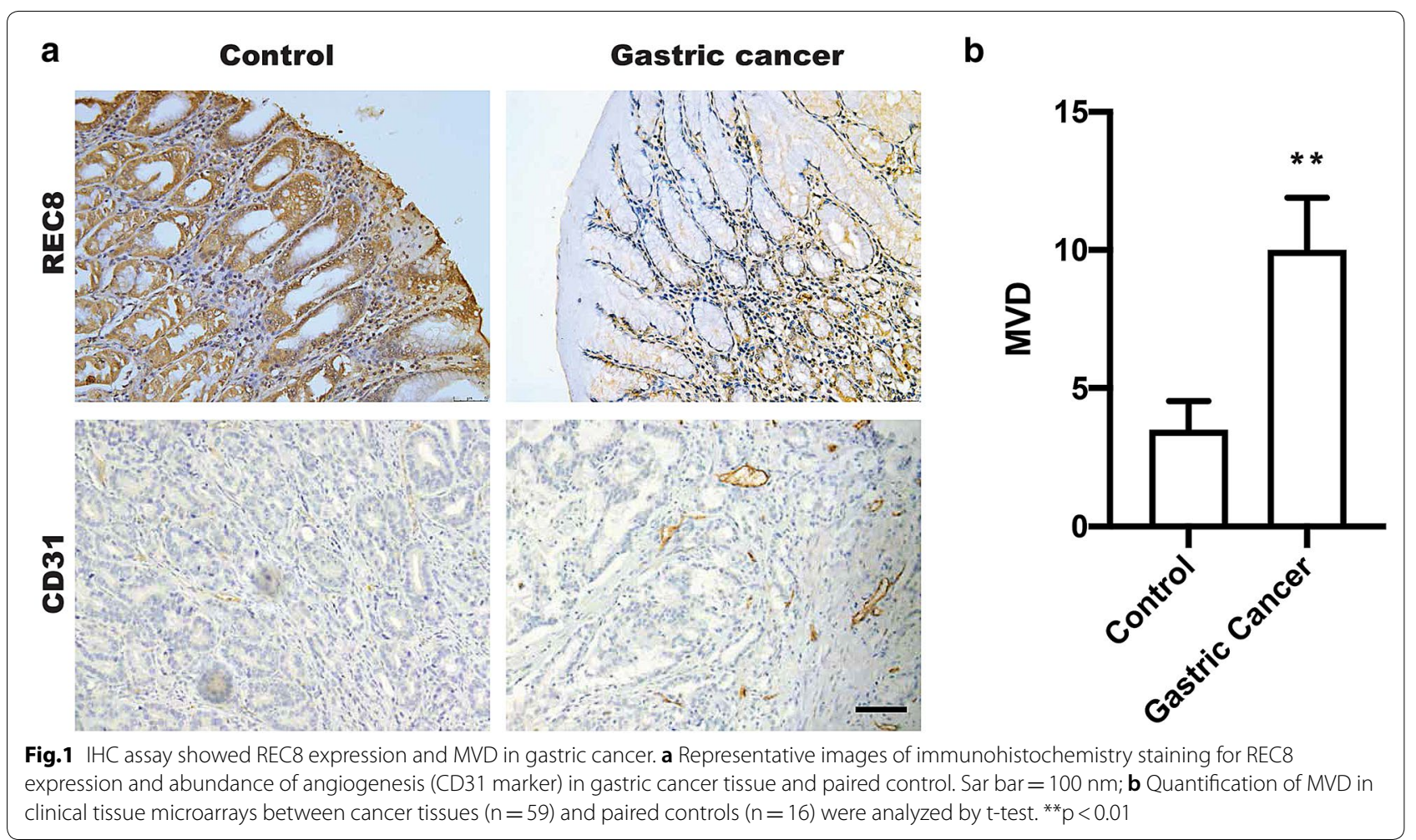

release of angiogenic factor VEGF. These data indicated that REC8 of gastric cancer cells inhibited tumor angiogenesis through VEGF.

\section{$\mathrm{NF}-\mathrm{KB}$ is required for REC8-mediated VEGF expression}

NF- $\mathrm{KB}$ has been reported to play an important role in VEGF expression [23, 24], which focused us to explore the possible effect of REC8 on NF-kB activity. As shown in Fig. 4a, silencing of REC8 expression in BGC823 and AGS-1 of gastric cancer cell promoted the phosphorylation of NF-kB p65 (Ser536), and no significance change of total NF- $\mathrm{kB}$ p65 was observed between shCTL and shREC8 (Additional file 1: Figure S1). To further explore the possibility that upregulation of VEGF transactivation resulting from REC8 depletion was caused by increased binding of binding of NF- $\mathrm{KB}$ to the VEGF promoter sequence, we utilized chromatin immunoprecipitation (ChIP). UCSC online software was used to identify a putative binding sequence, -86 GGGGCGGGCC -76 of $\mathrm{NF}-\mathrm{kB}$ in the promoter of $V E G F$ genes, which is similar to the NF- $\mathrm{kB}$ binding consensus sequence GGGRNNYYCC [25] (Fig. 4b). As shown in Fig. 4c, the baseline of the binding of $\mathrm{p} 65 \mathrm{NF}-\mathrm{kB}$ to the VEGF promoter region was dramatically increased in response to REC8 depletion.
Additionally, inhibition of NF- $\mathrm{kB}$ by NF- $\mathrm{B}$ inhibitor BAY11-7082 treatment could reverse the promotion of REC8 knockdown in VEGF expression, leading to attenuate the tube formation (Fig. 4d, e), implying involvement of NF- $\mathrm{KB}$ in REC8 tumor biological function.

\section{Discussion}

Up to now, no available reports about the function of REC8 in tumor angiogenesis. In this study, as shown in Fig. 4g, we are for the first time to demonstrate that knockdown of REC8 expression in BGC823 and AGS-1 of gastric cancer cells in vitro significantly contributed HUVECs recruitment through NF- $\mathrm{KB}$ mediated VEGF expression, thereby promoting tumor angiogenesis. Downregulation of REC8 was correlated with tumor angiogenesis in gastric cancer tissues. Moreover, slicing REC8 expression significantly promoted HUVECs migration and tube formation through regulating VEGF expression and secretion, Mechanically, REC8 interacted with NF- $\kappa B$ and inhibited NF- $\mathrm{kB}$ activity, leading to attenuate NF- $\mathrm{kB}$ nuclear translocation. These findings indicated the novel role of REC8 in tumor angiogenesis in gastric cancer and could be alterative therapy strategy 


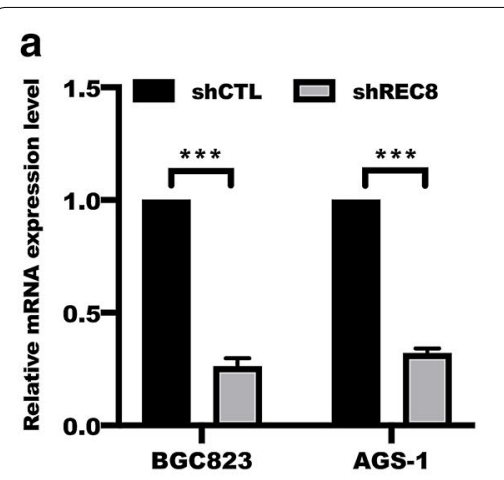

b

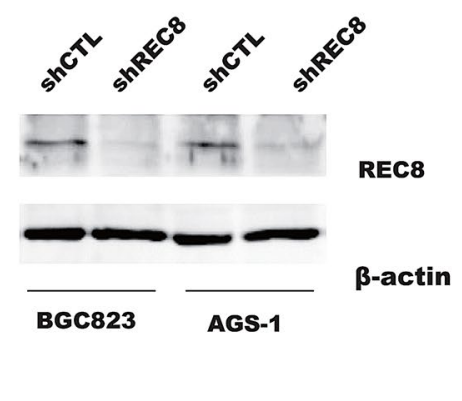

C

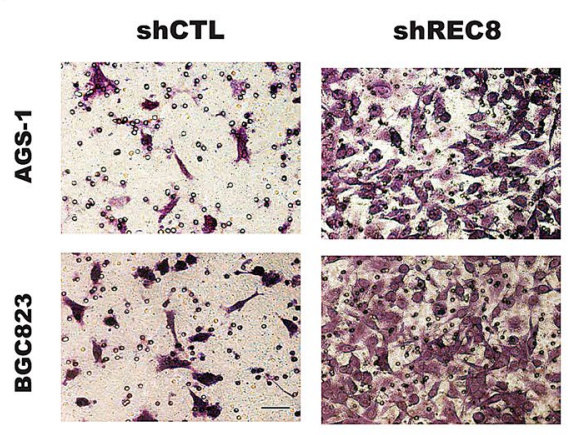

d

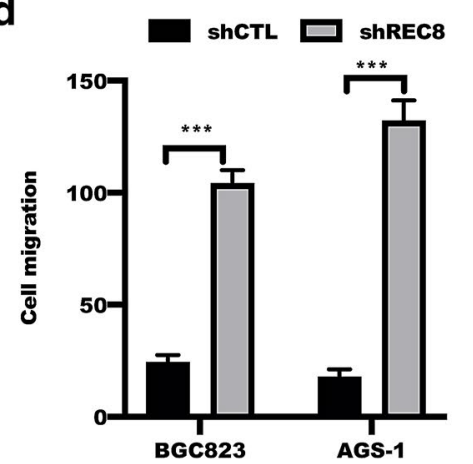

e

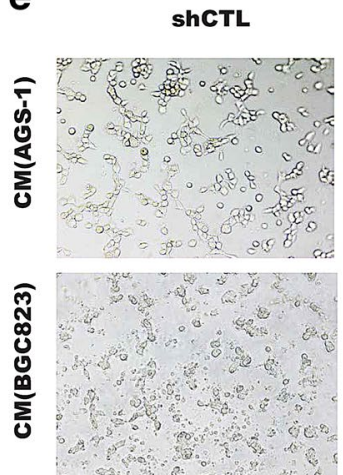

ShREC8

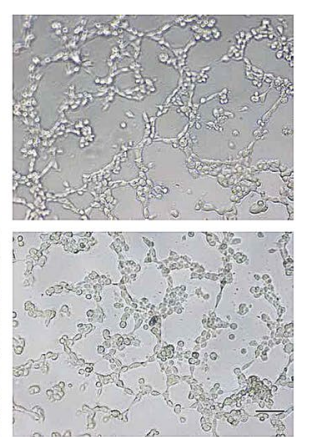

f

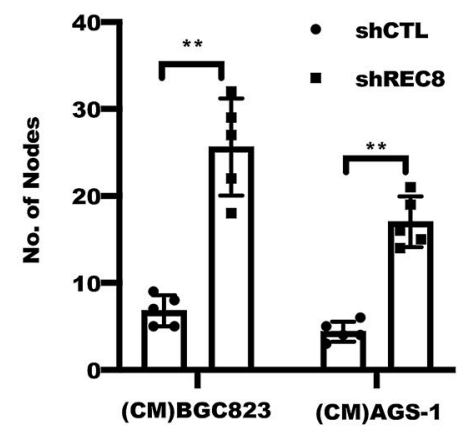

Fig.2 Gastric cancer cells with REC8 depletion promoted chemotactic migration and tube formation of endothelial cells. a Real-time and $\mathbf{b}$ western blotting were performed to examine the REC8 expression at mRNA and protein level. $\mathbf{c}$, d Conditional medium (CM) from BGC823 or AGS-1 cells infected with shCTL and shREC8 were used to be chemoattractant for HUVECs migration by transwell assays. ${ }^{* * *}$ p $<0.001$ versus shCTL group by t-tests. Sar bar=50um; e, f HUVECs were treated with conditional medium from BGC823 cells treated as indicated. Tube formation of HUVEC cells were visualized by phase contrast inverted microscope $(100 \times),{ }^{* *} \mathrm{p}<0.01$ versus shCTL group by t-tests

to recover REC8 expression and REC8-induced tumor angiogenesis.

REC8, a classically known to be a key component of the meiotic cohesion complex, played a critical role in chromosome dynamics during meiosis, including homology chromosome pairing, crossover recombination, and sister chromatid cohesion during meiosis [11, 26]. It is interesting to find that hypermethylation of the REC8 gene was uniquely more common in follicular thyroid cancer (FTC) and anaplastic thyroid cancer (ATC) [14]. In addition, the study by $\mathrm{Yu}$ et al. showed that a significant negative correlation between REC8 promoter methylation and mRNA expression by linear regression analysis, suggesting a prominent novel tumor suppressor gene that is epigenetically robustly targeted by the PI3K pathway in thyroid cancer [14]. Moreover, REC8 expression was associated with poor tumor prognosis in patients [27-29], which is not only attributed to cell growth and migration caused by REC8 reduction [30], but also promoted EGR1-mediated EMT [31]. In this study, we further demonstrated the novel function of REC8 in tumor angiogenesis by activation of NF-kB-VEGF-mediated recruitment of HUVECs and tube formation, inhibition of NF-kB by NF-kB inhibitor BAY11-7082 significantly revered the promotion of CM from REC8-depleted gastric cancer cells on tube formation, which would be required to confirm in vivo. What's more, REC8 interacted with NF- $\mathrm{kB}$, leading to inhibit NF- $\mathrm{kB}$ activity. Dynamic change NF- $\mathrm{kB}$ p65 activity is critical event in nucleus shuttle [32]. Depletion of REC8 expression in BGC823 cells drastically contributed the binding of NF- $\mathrm{kB}$ to VEGF promoter, leading VEGF transcription. However, in addition to VEGF, whether other pro-angiogenic factors, such as IL-6 et al., involved in REC8-mediated angiogenesis, whether REC8 regulated NF- $\mathrm{kB}$ activity in classical or non-classical pathway, and whether clinical trials with anti-angiogenic agents suppressed tumor angiogenesis in REC8-dependent manner, these unsolved issues remained to be addressed. 

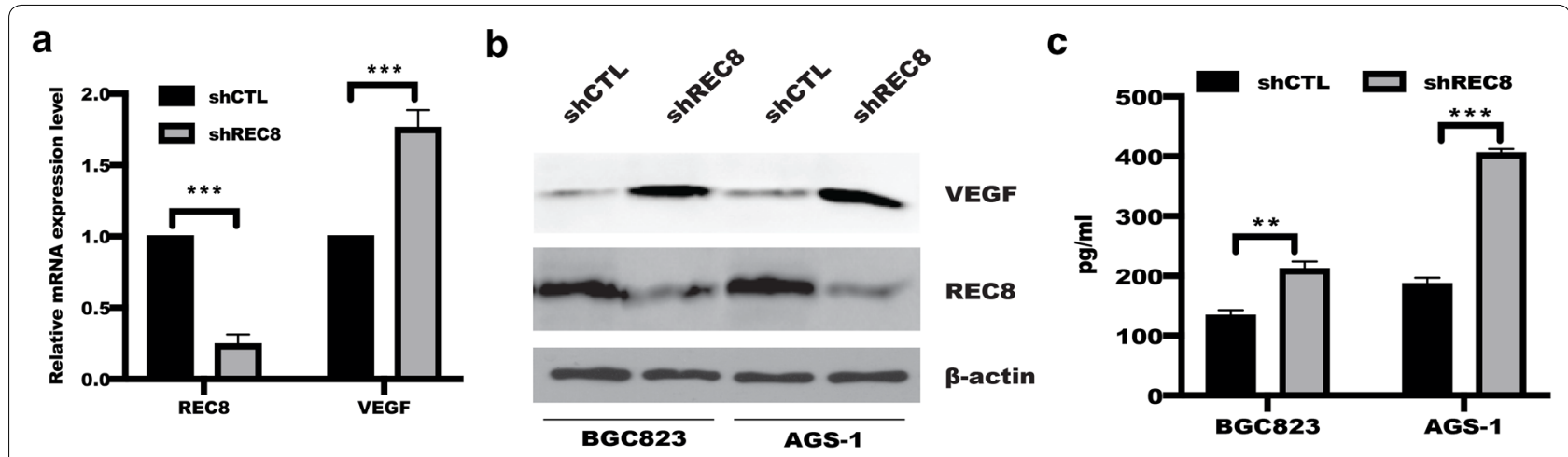

d

ShCTL

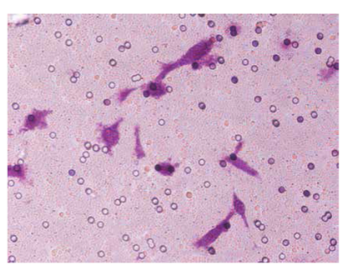

f

shCTL

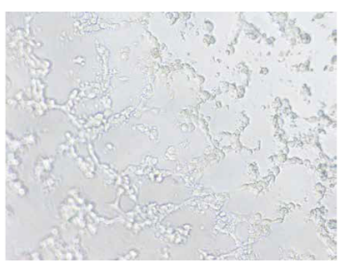

ShREC8

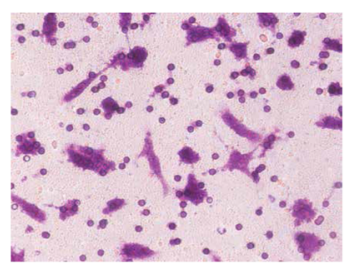

ShREC8

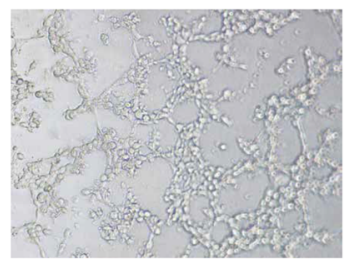

shREC8+anti-VEGF

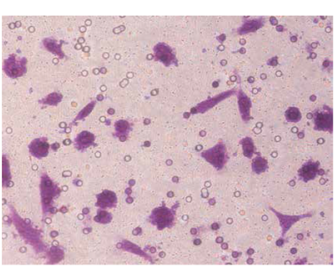

shREC8+anti-VEGF

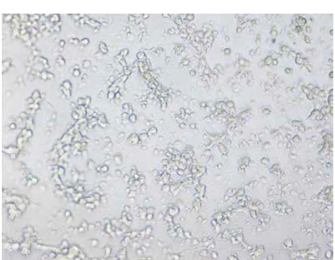

e

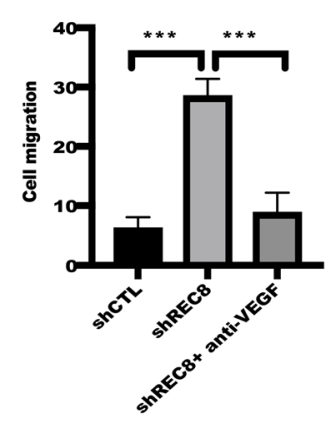

g

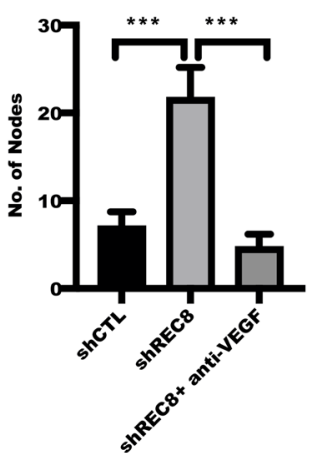

Fig.3 Depletion of REC8 enhanced HUVECs migration and tube formation through upregulation of VEGF in gastric cancer cells. a-c The mRNA and protein level of VEGF were analyzed by real-time qPCR, western blotting and ELISA in BGC823 and AGS-1 cells. ${ }^{* * *} p<0.001$, ${ }^{* *} p<0.01$ verus shCTL. $\mathbf{d}-\mathbf{g}$ Transwell assay and tube formation assay were employed to detect HUVEC migration and tube formation with or without neutralizing VEGF antibody in CM from indicated group in BGC823 cells. Quantification was performed to analyze the statistical significance. ${ }^{* *} \mathrm{p}<0.001$

In summary, in addition to the studies showed that the role of REC8 in tumor migration, invasion, cell proliferation, growth and apoptosis in gastric cancer [30]. Our study is the first time to unravel the role and mechanism of REC8 in tumor angiogenesis by regulation of NF-kBmediated VEGF expression in gastric cancer cells. Further studies are required to elucidate the role of REC8 in tumor immunology, tumor differentiation, tumor metabolism and remodeling tumor microenvironment.

\section{Conclusion}

The current study provided a novel insight into the functional role of REC8 in tumor angiogenesis, suggesting REC8 serves as a negative regulator in tumor angiogenesis through suppressing NF-kB-mediated VEGF, and demonstrating a novel function of REC8 in tumor progression. 


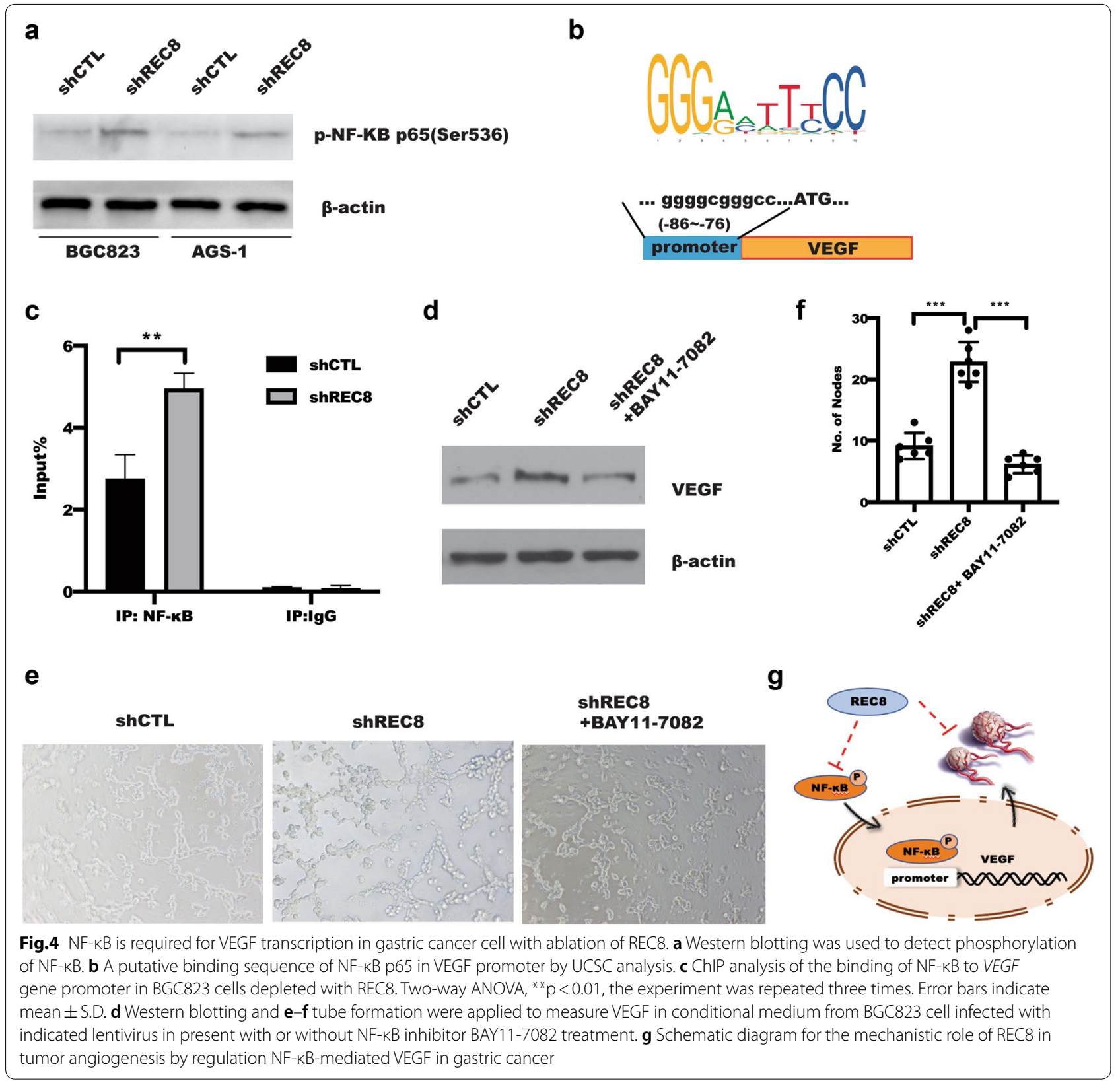

\section{Abbreviations}

CM: Conditional medium; ChIP: Chromatin immunoprecipitation; EMT: Epithelial-Mesenchymal Transition; ELISA: Enzyme Linked Immunosorbent Assay; GC: Gastric Cancer; HUVECs: Human Umbilical Vein Endothelial Cells; L: Interleukin; IP: Immunoprecipitation; IHC: Immunohistochemistry; MVD: Microvascular Density; NF-KB:: Nuclear factor-kB; PCR: Polymerase Chain Reaction; UCSC: University of California Santa Cruz; VEGF: Vascular Endothelial Growth Factor; WB: Western blotting.

\section{Acknowledgements}

None

\section{Authors' contributions}

ML and PSF conceived and designed the experiments, ML, WFX and MMS performed experiments and analyzed data, ML and WFX wrote the manuscript and revised manuscript. All authors read and approved the final manuscript. 


\section{Funding}

This research was supported by the Fundamental Research Funds for the Central Universities, the "New medicine" Foundation of University of Science and the Technology of China (grant number WK91 10000024) and Youth Fund Project of Anhui Provincial Cancer Hospital (grant number 2018YJQN009).

\section{Availability of data and materials}

The datasets generated during and/or analyses during the current study are available from the corresponding author on reasonable request.

\section{Ethics approval and consent to participate}

Not application.

\section{Consent for publication}

Not application.

\section{Competing interests}

The authors declared that there is no competing interests.

\begin{abstract}
Author details
${ }^{1}$ Anhui Provincial Hospital, Cheeloo College of Medicine, Shandong University, Jinan 250021, Shandong, China. ${ }^{2}$ The First Affiliated Hospital of USTC, Division of Life Sciences and Medicine, University of Science and Technology of China, Heifei 230031, Anhui, China. ${ }^{3}$ Department of Gastroenterology, Guangzhou Women and Children's Medical Center, Guangzhou Medical University, Guangzhou 510623, China. ${ }^{4}$ Guangzhou Institute of Pediatrics, Guangzhou Women and Children's Medical Center, Guangzhou Medical University, Guangzhou 510623, China. ${ }^{5}$ Department of Cancer Biology and Therapeutics, School of Pharmacy and Pharmaceutical Sciences, Cardiff University, Wales CF103AT, UK. ${ }^{6}$ Department of Oncology, Anhui Provincial Cancer Hospital, Hefei, Anhui 230001, P.R. China.
\end{abstract}

Received: 12 December 2019 Accepted: 7 September 2020 Published online: 21 September 2020

\section{References}

1. Wang FH, Shen L, Li J, Zhou ZW, Liang H, Zhang XT, et al. The Chinese Society of Clinical Oncology (CSCO): clinical guidelines for the diagnosis and treatment of gastric cancer. Cancer Commun. 2019;39(1):10.

2. Torre LA, Bray F, Siegel RL, Ferlay J, Lortet-Tieulent J, Jemal A. Global cancer statistics, 2012. CA Cancer J Clin. 2015;65(2):87-108.

3. Hanahan D, Weinberg RA. Hallmarks of cancer: the next generation. Cell. 2011;144(5):646-74.

4. Viallard C, Larrivee B. Tumor angiogenesis and vascular normalization: alternative therapeutic targets. Angiogenesis. 2017;20(4):409-26.

5. Ramjiawan RR, Griffioen AW, Duda DG. Anti-angiogenesis for cancer revisited: is there a role for combinations with immunotherapy? Angiogenesis. 2017;20(2):185-204

6. Chung AS, Lee J, Ferrara N. Targeting the tumour vasculature: insights from physiological angiogenesis. Nat Rev Cancer. 2010;10(7):505-14.

7. Chung AS, Ferrara N. Developmental and pathological angiogenesis. Annu Rev Cell Dev Biol. 2011;27:563-84.

8. Fu J, Ding $Y$, Huang $D$, Li H, Chen $X$. The retinoid $X$ receptor-selective ligand, LGD1069, inhibits tumor-induced angiogenesis via suppression of VEGF in human non-small cell lung cancer. Cancer Lett. 2007;248(1):153-63.

9. Zhang S, Cao Z, Tian H, Shen G, Ma Y, Xie H, et al. SKLB1002, a novel potent inhibitor of VEGF receptor 2 signaling, inhibits angiogenesis and tumor growth in vivo. Clin Cancer Res. 2011;17(13):4439-50.

10. Ferrara N. Vascular endothelial growth factor and the regulation of angiogenesis. Recent Prog Horm Res. 2000;55:15-35.

11. Nasmyth K. Disseminating the genome: joining, resolving, and separating sister chromatids during mitosis and meiosis. Annu Rev Genet. 2001;35:673-745.

12. Yoon SW, Lee MS, Xaver M, Zhang L, Hong SG, Kong YJ, et al. Meiotic prophase roles of Rec 8 in crossover recombination and chromosome structure. Nucleic Acids Res. 2016;44(19):9296-314.
13. Yu J, To KF, Liang QY. Epstein-Barr virus-driven promoter hypermethylated genes in gastric cancer. Hong Kong Med J. 2017;23 Suppl 5(4):17-22.

14. Liu D, Shen X, Zhu G, Xing M. REC8 is a novel tumor suppressor gene epigenetically robustly targeted by the PI3K pathway in thyroid cancer. Oncotarget. 2015;6(36):39211-24.

15. Zhao J, Liang Q, Cheung KF, Kang W, Lung RW, Tong JH, et al. Genome-wide identification of Epstein-Barr virus-driven promoter methylation profiles of human genes in gastric cancer cells. Cancer. 2013;119(2):304-12.

16. Xu W, Qian J, Zeng F, Li S, Guo W, Chen L, et al. Protein kinase Ds promote tumor angiogenesis through mast cell recruitment and expression of angiogenic factors in prostate cancer microenvironment. J Exp Clin Cancer Res. 2019;38(1):114.

17. Wu SY, Rupaimoole R, Shen F, Pradeep S, Pecot CV, Ivan C, et al. A miR192-EGR1-HOXB9 regulatory network controls the angiogenic switch in cancer. Nat Commun. 2016;7:11169.

18. Gao J, Zhao C, Liu Q, Hou X, Li S, Xing X, et al. Cyclin G2 suppresses Wnt/ beta-catenin signaling and inhibits gastric cancer cell growth and migration through Dapper1. J Exp Clin Cancer Res. 2018;37(1):317.

19. Lin X, Sun Q, Zhou L, He M, Dong X, Lai M, et al. Colonic epithelial mTORC1 promotes ulcerative colitis through COX-2-mediated Th17 responses. Mucosal Immunol. 2018;11(6):1663-73.

20. Xu W, Zhang Z, Zou K, Cheng Y, Yang M, Chen H, et al. MiR-1 suppresses tumor cell proliferation in colorectal cancer by inhibition of Smad3mediated tumor glycolysis. Cell Death Dis. 2017;8(5):e2761.

21. Vegran F, Boidot R, Michiels C, Sonveaux P, Feron O. Lactate influx through the endothelial cell monocarboxylate transporter MCT1 supports an NF-kappaB/IL-8 pathway that drives tumor angiogenesis. Cancer Res. 2011;71(7):2550-60.

22. Chatterjee S, Heukamp LC, Siobal M, Schottle J, Wieczorek C, Peifer M, et al. Tumor VEGF:VEGFR2 autocrine feed-forward loop triggers angiogenesis in lung cancer. J Clin Invest. 2013;123(4):1732-40.

23. Mu HQ, He YH, Wang SB, Yang S, Wang YJ, Nan CJ, et al. MiR-130b/TNFalpha/NF-kappaB/VEGFA loop inhibits prostate cancer angiogenesis. Clin Transl Oncol. 2019. https://doi.org/10.1007/s12094-019-02217-5.

24. Zhang B, Wang D, Ji TF, Shi L, Yu JL. Overexpression of IncRNA ANRIL upregulates VEGF expression and promotes angiogenesis of diabetes mellitus combined with cerebral infarction by activating NF-kappaB signaling pathway in a rat model. Oncotarget. 2017:8(10):17347-59.

25. Yang W, Xia Y, Cao Y, Zheng Y, Bu W, Zhang L, et al. EGFR-induced and PKCepsilon monoubiquitylation-dependent NF-kappaB activation upregulates PKM2 expression and promotes tumorigenesis. Mol Cell. 2018;69(2):347.

26. Watanabe Y, Nurse P. Cohesin Rec8 is required for reductional chromosome segregation at meiosis. Nature. 1999;400(6743):461-4.

27. Laird PW. The power and the promise of DNA methylation markers. Nat Rev Cancer. 2003;3(4):253-66.

28. Furuta J, Nobeyama Y, Umebayashi Y, Otsuka F, Kikuchi K, Ushijima T. Silencing of peroxiredoxin 2 and aberrant methylation of $33 \mathrm{CpG}$ islands in putative promoter regions in human malignant melanomas. Cancer Res. 2006;66(12):6080-6.

29. Okamoto Y, Sawaki A, Ito S, Nishida T, Takahashi T, Toyota M, et al. Aberrant DNA methylation associated with aggressiveness of gastrointestinal stromal tumour. Gut. 2012;61(3):392-401.

30. Yu J, Liang Q, Wang J, Wang K, Gao J, Zhang J, et al. REC8 functions as a tumor suppressor and is epigenetically downregulated in gastric cancer, especially in EBV-positive subtype. Oncogene. 2017;36(2):182-93.

31. Zhao J, Geng L, Duan G, Xu W, Cheng Y, Huang Z, et al. REC8 inhibits EMT by downregulating EGR1 in gastric cancer cells. Oncol Rep. 2018;39(4):1583-90.

32. Zou K, Wang Y, Hu Y, Zheng L, Xu W, Li G. Specific tumor-derived CCL2 mediated by pyruvate kinase $\mathrm{M} 2$ in colorectal cancer cells contributes to macrophage recruitment in tumor microenvironment. Tumour Biol. 2017;39(3):1010428317695962.

\section{Publisher's Note}

Springer Nature remains neutral with regard to jurisdictional claims in published maps and institutional affiliations. 